\title{
In Situ STEM Observations of Elemental Segregation in Phase Change Material GST Under Electrical and Thermal Stress
}

Ho Leung Chan ${ }^{1}$, Matthew Mecklenburg ${ }^{2}$, William Hubbard ${ }^{3}$, Jared Lodico ${ }^{4}$, Brian Zutter ${ }^{1}$ and B. C. Regan $^{4}$

${ }^{1}$ University of California, Los Angeles, Los Angeles, California, United States, ${ }^{2}$ The Aerospace Corporation, United States, ${ }^{3}$ NanoElectronic Imaging, Inc., Los Angeles, California, United States, ${ }^{4}$ Department of Physics and Astronomy, University of California, Los Angeles, California 90095, United States, United States

$\mathrm{Ge}_{2} \mathrm{Sb}_{2} \mathrm{Te}_{5}$ (GST) is a promising candidate material for phase change memory (PCM) because its electrical resistivity changes dramatically between its amorphous and crystalline phases [1]. Understanding the different failure modes in PCM is important for increasing device stability and lifetime. Electric fields and thermal gradients produced by programing pulses induce elemental segregation in PCM $[2,3]$, which can lead to device failure. Here, we use scanning transmission electron microscopy (STEM) and energy dispersive $\mathrm{x}$-ray spectroscopy (EDS) to study elemental segregation in PCM under electrical and thermal stress.

Using e-beam lithography, we pattern a TiN heater, TiN electrodes, and a GST bridge on an electron transparent $\mathrm{Si}_{3} \mathrm{~N}_{4}$ membrane (Fig. 1). Both the TiN $(30 \mathrm{~nm})$ and the GST $(50 \mathrm{~nm})$ are deposited with magnetron sputtering. To prevent the GST from oxidizing during heating, we use atomic layer deposition (ALD) to cap the entire device with $20 \mathrm{~nm}$ of $\mathrm{Al}_{2} \mathrm{O}_{3}$. The on-chip heater and electrodes allow for independent heating and biasing of the GST in situ. The GST bridge is surrounded by the TiN heater on three sides to minimize temperature gradients. As deposited, the GST is amorphous (Fig. 1B). Powering up the TiN heater crystallizes the GST to its fcc and hexagonal phases (Fig. $1 \mathrm{C}$ and D). Driving $100 \mu \mathrm{A}$ through the GST bridge while simultaneously raising the heater power causes some of the GST to electromigrate (Fig. 1D).

We acquire EDS maps (Fig. 2) using an Oxford X-MaxN 100 TLE EDS detector on an FEI Titan S/TEM operating at $80 \mathrm{kV}$. The EDS maps in Figs. 2 A and B correspond to the GST states in Figs. $1 \mathrm{~B}$ and D, respectively. Data outside of the GST bridge are excluded in the EDS maps. The line profiles (Fig. 2C) are measured from the left to the right of each frame and averaged in the vertical direction. As fabricated, the three elements are uniformly distributed across the GST bridge. The stoichiometry is measured to be within $6 \%$ of the nominal 2-2-5. After 5 minutes with $210 \mu \mathrm{W}$ dissipation in the heater and $100 \mu \mathrm{A}$ passing from the right to left through the GST, some elemental segregation is observed. This heater power puts the GST near its $420 \mathrm{~K}$ amorphous-fcc transition temperature. Regions on the anode with an overall material migration exhibits no detectable elemental segregation, so they are masked out in the EDS maps. Of the three constituent elements in GST, tellurium appears to migrate the most, with significant depletion in the cathode (dark contrast in Fig. 2B). Germanium and antimony move in the opposite direction and accumulate in the cathode, as seen in the line profiles. These observations of the polarity dependence of elemental segregation are consistent with those reported previously [3, 4]. Further study will uncover the effects of elemental segregation, decouple the effects of electrical and thermal stress, and ultimately contribute to improving PCM reliability. 
A

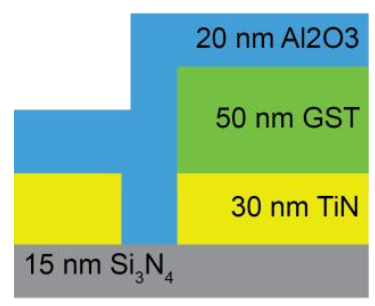

B
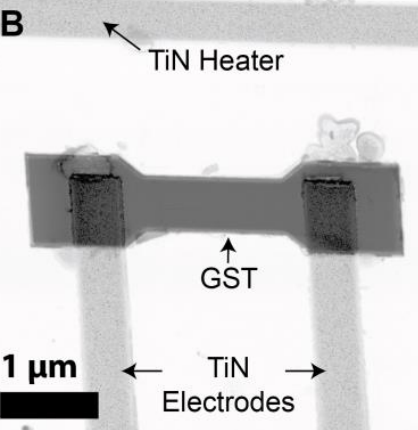

C

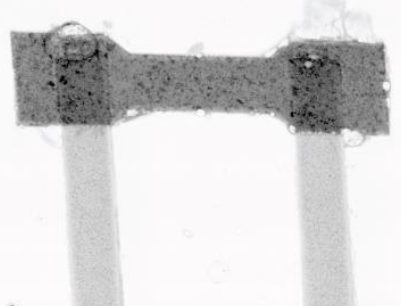

D

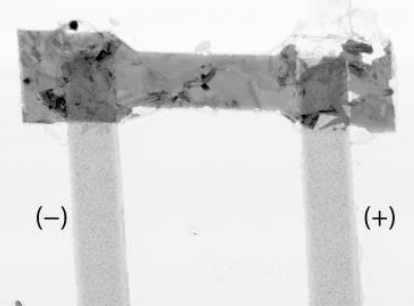

Figure 1. Figure 1. Schematic of device cross-section (A) and time series of BF STEM images of a GST bridge undergoing phase change (B-D). The device consists of an electron transparent Si3N4 membrane, a TiN heater, a pair of TiN electrodes, a GST bridge, and a layer of ALD A12O3. The resistance of the GST at the three different crystallization stages is $3.9 \mathrm{G} \Omega(\mathrm{B}), 18.6 \mathrm{M} \Omega(\mathrm{C})$, and $8.2 \mathrm{k} \Omega(\mathrm{D})$. The (+) and $(-)$ signs in (D) indicate the bias voltage polarity.

A
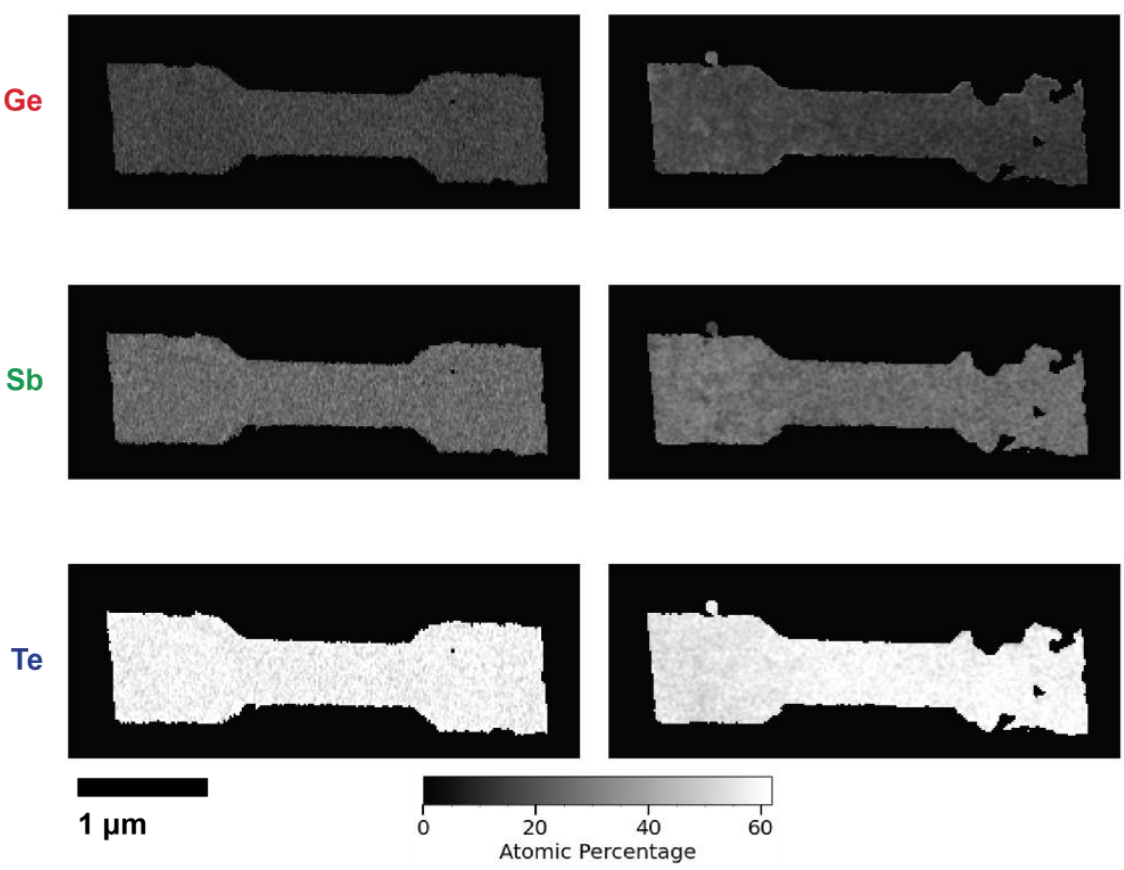

B
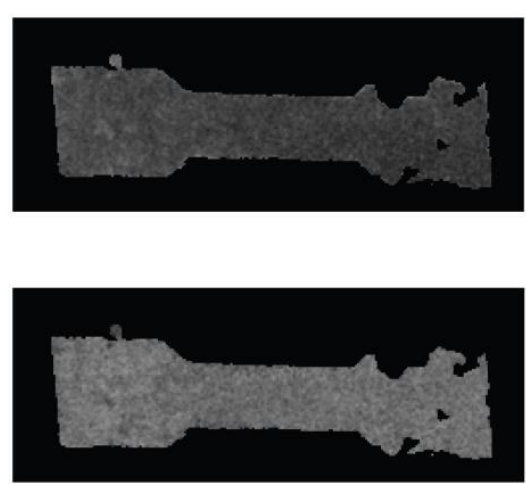
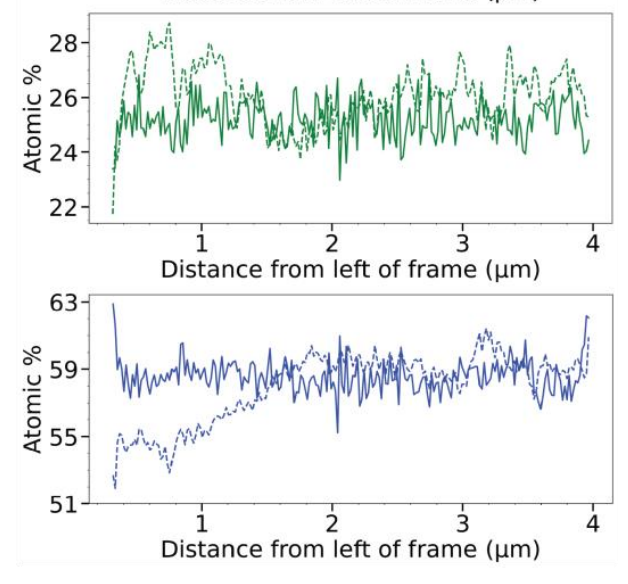

Figure 2. Figure 2. EDS elemental composition maps of $\mathrm{Ge}, \mathrm{Sb}$, and Te before (A) and after (B) thermal and electrical bias on the device, and line scans of atomic concentration $(C)$. The regions outside of the GST bridge are masked, and the line scans are vertically averaged over the entire device. The before- and after- line profiles are shown as solid and dashed lines, respectively.

\section{References}

[1] GW Burr et al., Journal of Vacuum Science \& Technology B 28 (2010), p. 223-262.

[2] Y Xie et al., Adv. Mater. 30 (2018), 1705587.

[3] P Yeoh et al., Appl. Phys. Lett. 114 (2019), 163507.

[4] TY Yang et at., Appl. Phys. Lett. 95 (2009), 032104.

[5] This work was supported by SRC NMP 2872.001, NSF STC award DMR-1548924 (STROBE), and NSF award DMR-1611036. 